\title{
Quasiballistic Thermal Transport from Nanoscale Heaters and the Role of the Spatial Frequency
}

\author{
Xiangwen Chen, ${ }^{1, \dagger}$ Chengyun Hua, ${ }^{2, \dagger}$ Hang Zhang, ${ }^{3}$ Navaneetha K. Ravichandran, ${ }^{4}$ and \\ Austin J. Minnich ${ }^{1, *}$ \\ ${ }^{1}$ Division of Engineering and Applied Science, California Institute of Technology, Pasadena, California 91125, \\ USA \\ ${ }^{2}$ Oak Ridge National Laboratory, Oak Ridge, Tennessee 37831, USA \\ ${ }^{3}$ Institute of Engineering Thermophysics, Chinese Academy of Sciences, Beijing 100190, China \\ ${ }^{4}$ Department of Physics, Boston College, Chestnut Hill, Massachusetts 02467, USA
}

(Received 24 August 2017; revised manuscript received 24 May 2018; published 29 November 2018)

\begin{abstract}
Quasiballistic heat conduction from nanoscale heat sources of size comparable to phonon mean free paths has recently become of intense interest both scientifically and for its applications. Prior work has established that, in the quasiballistic regime, the apparent thermal properties of materials depend both on intrinsic mechanisms and the characteristics of the applied thermal gradient. However, many aspects of this regime remain poorly understood. Here, we experimentally study the thermal response of crystals to large thermal gradients generated by optical heating of nanoline arrays. Our experiments reveal the key role of the spatial frequencies and Fourier series amplitudes of the heating profile for thermal transport in the quasiballistic regime, in contrast to the conventional picture that focuses on the geometric dimensions of the individual heaters. Our work provides the insight needed to rationally mitigate local hot spots in modern applications by manipulating the spatial frequencies of the heater patterns.
\end{abstract}

DOI: 10.1103/PhysRevApplied.10.054068

\section{INTRODUCTION}

Heat conduction by phonons is of both fundamental and practical importance, playing a key role in modern applications ranging from thermoelectrics [1-5] to electronic devices [6-10]. The limiting regimes of heat conduction are well understood. If thermal gradients occur over length scales much longer than mean free paths (MFPs), heat conduction occurs by diffusion as described by Fourier's law. In the opposite limit of an extreme thermal gradient over which no scattering occurs, heat conduction occurs by phonon radiation in an exact analogy to blackbody radiation [11]. The intermediate quasiballistic regime, in which some phonons are ballistic but some undergo scattering events, has recently become of intense interest [12-23]. This regime occurs in practice much more frequently than the completely ballistic regime because phonons possess a very broad MFP spectrum [24] and often not all phonons are ballistic for experimentally achievable heating length scales.

Koh and Cahill first reported variations of thermal conductivity with modulation frequency in time-domain thermoreflectance (TDTR) experiments that they attributed to

\footnotetext{
*aminnich@caltech.edu

${ }^{\dagger}$ These authors contributed equally to this work.
}

quasiballistic transport [13]. Subsequently, a number of works reported observations of quasiballistic effects in systems including lithographically patterned nanoline arrays on sapphire [14], silicon at cryogenic temperatures [15] as well as at room temperature [18,19], and thin silicon membranes at room temperature [25]. A recent work reported the existence of a collective-diffusive regime [20], again involving nanolines with variable periods. These effects have been used to map the spectral thermal conductivity of crystals $[16,17]$. Meanwhile, numerous models have been proposed to explain the various observations, including two-channel models with ballistic and diffusive modes $[26,27]$, approximate solutions of the Boltzmann transport equation (BTE) [23,28], a superdiffusive formalism [21,22], and numerical methods [29].

Despite these prior studies, many aspects of the quasiballistic regime remain poorly understood, with prior works drawing contradictory conclusions. For example, Hu et al. [16] and Zeng et al. [17] report large and monotonic increases in thermal resistance as the dimensions of individual heaters in patterned arrays become smaller than MFPs. However, other works suggest that size effects should not play a role in closely spaced patterned heaters due to the lack of in-plane thermal gradient [30]. Overall, a comprehensive understanding of the thermal response of solids to large temperature gradients is lacking, impacting 
efforts to enhance heat dissipation in modern devices that possess nanoscale heat sources.

Here, we experimentally study quasiballistic transport in crystals generated by optically heating metallic nanoline arrays. Our experiments reveal the key role of the spatial frequencies and Fourier series amplitudes of the heating profile for thermal transport in the quasiballistic regime, in contrast to the conventional picture in which the characteristic dimensions of the individual heaters play the central role. In addition, our work provides the insight needed to rationally mitigate local hot spots in modern applications by manipulating the spatial frequencies of the heater patterns.

\section{METHODS}

\section{A. TDTR measurements and fitting model}

We use two-tint TDTR [31] to study heat conduction in $c$-sapphire with patterned aluminum line arrays as transducer. Briefly, the pump pulse train, at a 76-MHz repetition rate and wavelength of $785 \mathrm{~nm}$, is amplitude-modulated at $\eta_{0}$ and directed to the sample to provide a heat impulse. The change in reflectance of the aluminum lines due to the temperature change is detected by a reflected probe beam with wavelength near $785 \mathrm{~nm}$ but spectrally distinct from the pump using sharp-edged optical filters. Sapphire is chosen as the substrate as it is transparent to the 785-nm laser used in the experiments so that only the aluminum lines absorb the incident pump light, as shown in Fig. 1(a). The pump and probe beams are fixed with $1 / e^{2}$ diameters of 30 and $10 \mu \mathrm{m}$, respectively. Both beam sizes are measured using a home-built two-axis knife-edge beam profiler. We use a mechanical delay line with up to $14 \mathrm{~ns}$ of total delay time. All the measurements are performed in an optical cryostat (JANIS ST-500) under high vacuum of $10^{-6}$ Torr. The experimental data consist of in-phase and out-of-phase signal versus delay time as measured by a rf lock-in amplifier (Zurich Instruments HF2LI). The signal is converted into amplitude and phase for fitting and presentation, as shown in Figs. 1(e) and 1(f).

To fit the nanoline data, we assume that both the pump and probe spot sizes are much larger than the linewidth and period, allowing us to consider the nanoline heating profile as spatially periodic along the $x$ axis, as shown in Figs. 1(a) and 1(b). The $y$-axis direction is considered as infinite. As a result, the heat transport can be described using a 2D model in Cartesian coordinates. The square wave profile of the lines is described mathematically by imposing a squarewave heating at the $\mathrm{Al}$ transducer layer surface and setting the in-plane thermal conductivity of the transducer layer $\kappa_{r}=0$. The solution then follows exactly the same derivation as given in Refs. [32] and [33]. Further details of the fitting model are available in Ref. [17]. The only difference between the present model and this prior model is that we allow some of the frequency components of the
TDTR signal to have different thermal properties rather than forcing all frequency components to have the same value of the fitting parameter as in the traditional model.

\section{B. Sample fabrication and characterization}

The sample consists of aluminum nanoline arrays with an area of $60 \times 60 \mu \mathrm{m}^{2}$ fabricated on $c$-sapphire substrates as shown schematically in Fig. 1(a), using a standard electron-beam lithography and lift-off process. The width of the lines $w$ varies between $50 \mathrm{~nm}$ and $1.5 \mu \mathrm{m}$, while the period of line array $L$ ranges from around 1.5 to 4.5 times the corresponding linewidth; the duty cycle is defined as $w / L$. Owing to the spatial periodicity of the heating pattern, it can be represented as a Fourier series with discrete spatial-frequency components of a square wave as shown in Fig. 1(b). Single-side polished $c$-sapphire substrates from University Wafers are first cleaned in Nanostrip, followed by sonication in acetone and isopropyl alcohol (IPA), and then rinsed with IPA and dried with dry $\mathrm{N}_{2}$. Approximately $100-\mathrm{nm}$ poly(methyl methacrylate) (PMMA) is spun on the substrate, followed by baking at $180^{\circ} \mathrm{C}$ for $5 \mathrm{~min}$. After cooling, a conductive layer of AquaSave (Mitsubishi Rayon Co. Ltd.) is spun on the resist as an anticharging layer for e-beam lithography. The resist is exposed to the electron beam in a Leica/Vistec EBPG $5000+$ electron beam writer operating at $100-\mathrm{kV}$ accelerating voltage and a 5-nm spot size. After patterning, the AquaSave is removed with a rinse of DI water and then the sample is dried with $\mathrm{N}_{2}$. Development is performed at $4{ }^{\circ} \mathrm{C}$ in a 1:3 mixture of methyl isobutyl ketone (MIBK) and IPA for $65 \mathrm{~s}$ and terminated in IPA for $1 \mathrm{~min}$. The patterns are subjected to a brief dose of oxygen plasma to clean the PMMA residues after development. Al film $42 \mathrm{~nm}$ thick is deposited with a Lesker LAB Line $E$-beam evaporator at a pressure of $7 \times 10^{-7}$ Torr. Lift-off is performed in dichloromethane at room temperature for $15 \mathrm{~min}$.

The dimensions of aluminum line arrays, including the linewidth, period, and height, are characterized by atomic force microscopy (AFM) (Bruker Dimension ICON) and scanning electron microscopy (SEM). For smaller lines, the cross section is also characterized by transmission electron microscopy (TEM) (FEI Tecnai TF-20). The TEM samples are prepared with a standard focused ion beam (FIB) lift-out technique and FIB milling for thinning to $<100 \mathrm{~nm}$ after TDTR measurements are performed. AFM and cross-section TEM images of the fabricated lines are presented in Figs. 1(c) and 1(d). The linewidth is computed using AFM measurements corrected for the tip radius and these measurements are confirmed with the cross-section TEM measurements for select lines. Accurate determination of linewidths is essential as the TDTR measurements are exceedingly sensitive to this parameter; see Sec. 4 and Fig. S8 in the Supplemental Material [34] 
(a)

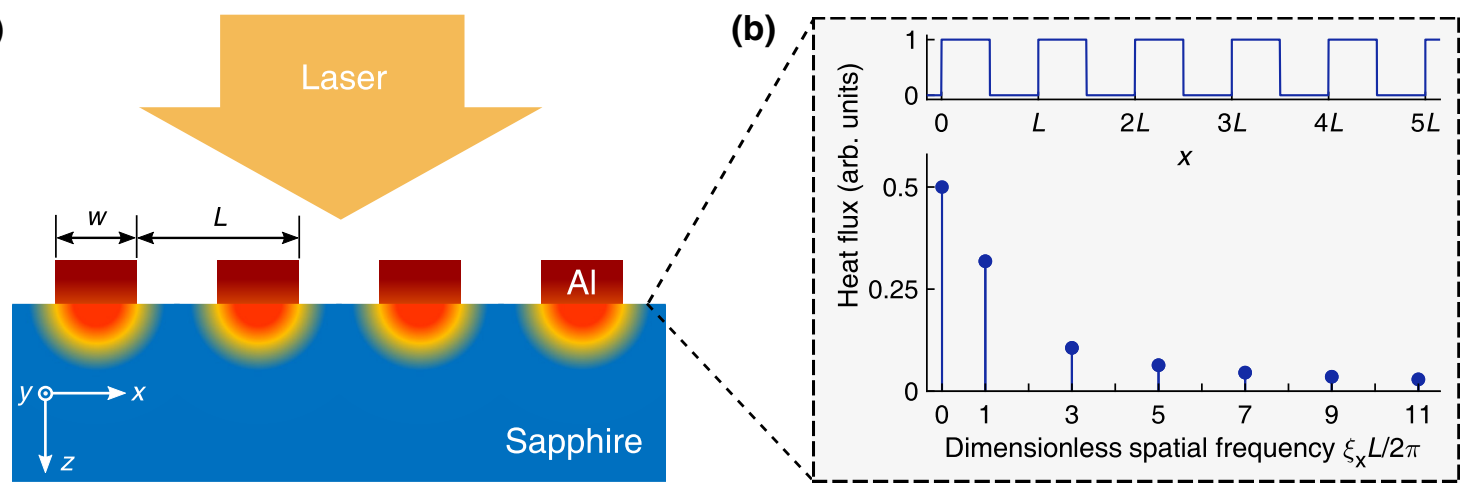

(c)

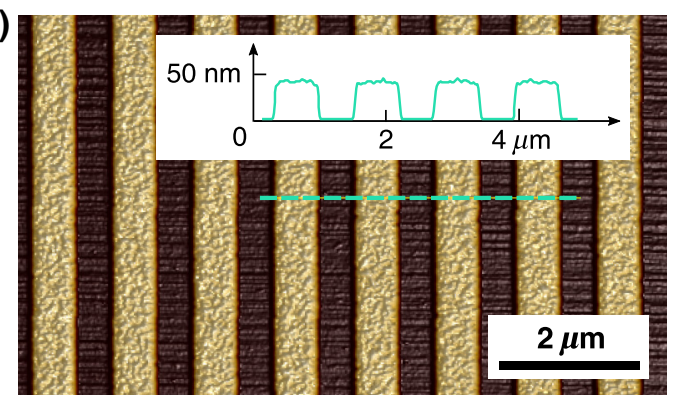

(e)

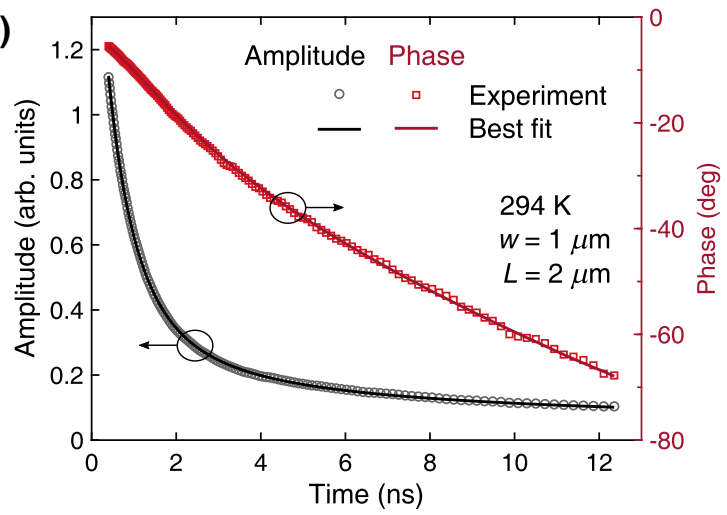

(d)

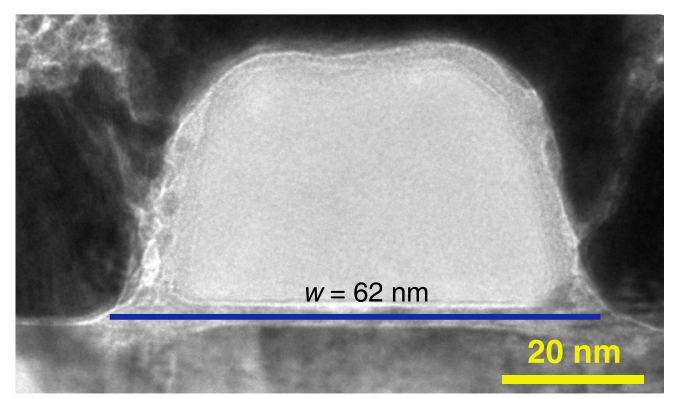

(f)

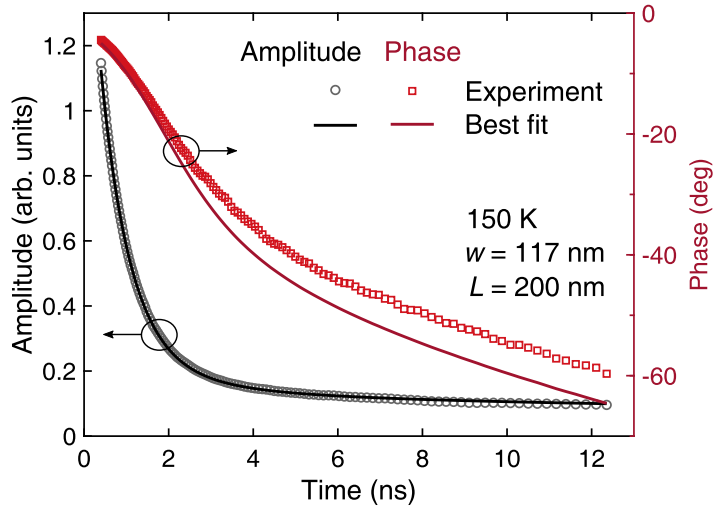

FIG. 1. Schematic of experimental geometry and example data from nanoline arrays. (a) Schematic of sample geometry. (b) Spatial heating profile on the substrate in real space (top) and versus spatial frequency (bottom). In the experiment, the probe beam that measures the thermal response has an identical geometry. (c) Representative AFM topography of 627-nm line arrays with a period of $1.2 \mu \mathrm{m}$. The dashed green line indicates the cross section used for the height profile shown in the inset. (d) TEM cross-section profile of a 62-nm-width line. (e),(f) Representative TDTR amplitude and phase experimental data and best fit using a standard heat diffusion model [16] on line arrays at (e) 5.3-MHz modulation frequency, $w=1 \mu \mathrm{m}$, and $L=2 \mu \mathrm{m}$ at $294 \mathrm{~K}$ and (f) $3.1 \mathrm{MHz}, w=117 \mathrm{~nm}$, and $L=200 \mathrm{~nm}$ at $150 \mathrm{~K}$. In (e), the best-fit curve matches the data and yields a fitted thermal conductivity and interface conductance of $38 \mathrm{~W} \mathrm{~m}^{-1} \mathrm{~K}^{-1}$ and $196 \mathrm{MW} \mathrm{m}^{-2} \mathrm{~K}^{-1}$, respectively. In (f), the phase fitting is poor. In this situation, fitting to amplitude [16] or phase [17] will give different results.

\section{Spectral BTE calculation}

Thermal transport in the nanoline array system is described by the two-dimensional spectral BTE under the relaxation time approximation (RTA),

$$
\begin{aligned}
\frac{\partial g_{\omega}}{\partial t}+v_{\omega, x} \frac{\partial g_{\omega}}{\partial x}+v_{\omega, z} \frac{\partial g_{\omega}}{\partial z}= & -\frac{g_{\omega}+f_{0}\left(T_{0}\right)-f_{0}(T)}{\tau_{\omega}} \\
& +\frac{Q_{\omega}(x, z, t)}{4 \pi}
\end{aligned}
$$

$$
f_{0}(T)=\frac{1}{4 \pi} \hbar \omega D(\omega) f_{\mathrm{BE}}(T) \approx f_{0}\left(T_{0}\right)+\frac{1}{4 \pi} C_{\omega} \Delta T,
$$

where $g_{\omega}=\hbar \omega D(\omega)\left[f_{\omega}(x, t, \mu)-f_{0}\left(T_{0}\right)\right]$ is the deviational distribution function; $f_{0}=f_{0}(x, t)$ is the equilibrium distribution function; $\mu=\cos (\theta)$ is the directional cosine; $v_{\omega, x}$ and $v_{\omega, z}$ are the phonon group velocities in the $x$ (inplane) and $z$ (cross-plane) directions; and $\tau_{\omega}$ is the phonon relaxation time. For a line array heating pattern, $Q_{\omega}(x, z, t)$, 
the spectral volumetric heat generation, is formulated as follows

$$
Q_{\omega}(x, z, t)=Q_{\omega}(z, t) \operatorname{rect}(x),
$$

where $\operatorname{rect}(x)$ represents a square wave.

Assuming a small temperature rise, $\Delta T=T-T_{0}$, relative to a reference temperature, $T_{0}$, the equilibrium distribution is proportional to $\Delta T$, as shown in Eq. (2). Here, $\hbar$ is the reduced Planck constant, $\omega$ is the phonon frequency, $D(\omega)$ is the phonon density of states, $f_{\mathrm{BE}}$ is the Bose-Einstein distribution, and $C_{\omega}=\hbar \omega D(\omega)\left(\partial f_{\mathrm{BE}} / \partial T\right)$ is the mode specific heat. The volumetric heat capacity is then given by $C=\int_{0}^{\omega_{m}} C_{\omega} d \omega$ and the Fourier thermal conductivity $\kappa=\int_{0}^{\omega_{m}} \kappa_{\omega} d \omega$, where $\kappa_{\omega}=\frac{1}{3} C_{\omega} v_{\omega} \Lambda_{\omega}$ and $\Lambda_{\omega}=\tau_{\omega} v_{\omega}$ is the phonon MFP. To close the problem, energy conservation is used to relate $g_{\omega}$ to $\Delta T$, given by

$$
\iint_{0}^{\omega_{m}}\left[\frac{g_{\omega}(x, t)}{\tau_{\omega}}-\frac{1}{4 \pi} \frac{C_{\omega}}{\tau_{\omega}} \Delta T(x, t)\right] d \omega d \Omega=0,
$$

where $\Omega$ is the solid angle and $\omega_{m}$ is the cutoff frequency. Note that summation over phonon branches is implied without an explicit summation sign whenever an integration over phonon frequency or MFP is performed.

Since a typical laser spot size is much larger (at least 10-100 times larger) than the linewidth/period of the line array, the in-plane direction parallel to the lines (the $y$-axis direction) is assumed to be infinite. As a result, heat transport in the line array is reduced to a two-dimensional heat transfer problem as shown in Fig. 1(a). The in-plane direction perpendicular to the lines is also assumed to be infinite and we neglect any effect of the finite pump size. Therefore, a Fourier transform can be applied to the in-plane direction perpendicular to the lines and Eq. (1) becomes

$$
\begin{aligned}
& \frac{\partial \tilde{g}_{\omega}}{\partial t}+i \xi_{x} \tilde{g}_{\omega} v_{\omega, x}+v_{\omega, z} \frac{\partial \tilde{g}_{\omega}}{\partial z}=-\frac{\tilde{g}_{\omega}}{\tau_{\omega}}+\frac{1}{4 \pi} C_{\omega} \Delta \tilde{T}\left(\xi_{x}, z, t\right) \\
& +\frac{Q_{\omega}(z, t)}{4 \pi} \sum_{n=-\infty}^{+\infty} \frac{2 \sin (\pi n w / L)}{n}
\end{aligned}
$$

where $\sum_{n=-\infty}^{+\infty}[2 \sin (\pi n w / L) / n]$ is the Fourier transform of a square wave and $\xi_{x}$ is the Fourier variable in the $x$ direction. $\xi_{x}$ is discrete and takes the value of $2 \pi n / L$, where $L$ is the period length and $n$ is an integer.

The calculation can be divided into three parts: the transducer layer, substrate, and interface. In the transducer layer, the transport is considered only in the cross-plane direction. Therefore, $\xi_{x}=0$. The BTE in the transducer layer can be reformulated as a Fredholm integral equation of the second kind and solved using the method of Ref. [35]. The solution in the substrate can be obtained using the multidimensional Green's function to the BTE [36]. The solutions in the two layers depend on each other through the interface conditions that enforce conservation of heat flux. The detailed discussion and derivation exactly follow those given in Ref. [37] excepting the use of the multidimensional Green's function in this work. The dispersion and relaxation times for sapphire are approximated from first-principles calculations by Lucas Lindsay for Si [38]; the relaxation times are divided by 4 to more closely match the thermal conductivity of sapphire.

\section{RESULTS AND DISCUSSION}

\section{A. Observing quasiballistic heat conduction}

Experimental amplitude and phase data from the lock-in amplifier for $1-\mu \mathrm{m}$ lines with $50 \%$ duty cycle at room temperature are given in Fig. 1(e). We fit these data using a traditional multilayer heat diffusion model $[16,32,33]$ with the substrate thermal conductivity and interface conductance between the lines and substrate as fitting parameters. We see in Fig. 1(e) that the fitting quality is excellent and yields a thermal conductivity of $38 \mathrm{~W} \mathrm{~m}^{-1} \mathrm{~K}^{-1}$, which is in good agreement with the literature value for sapphire [39, 40]. In contrast, Fig. 1(f) presents the amplitude and phase data for $117-\mathrm{nm}$ lines at $150 \mathrm{~K}$. Although the fitted amplitude reasonably matches the experimental data, the phase fit is quite poor. This discrepancy indicates that heat diffusion theory is failing to describe key aspects of the transport dynamics, complicating the interpretation of the data.

\section{B. Interpreting the TDTR measurement}

The Boltzmann transport equation is the most rigorous formalism to analyze the measured data, but a simplified model can provide insight into the experimental data without requiring extensive microscopic input. To identify this model, we examine the TDTR signal more closely. The transfer function $Z(t)$ that relates the measured amplitude and phase of the surface temperature to a spatially periodic input surface heat flux is given by

$$
Z(t)=\sum_{m=-\infty}^{-\infty} \sum_{n=-\infty}^{-\infty} H\left(\eta_{0}+m \eta_{s}, \xi_{x, n}\right) e^{i m \eta_{s} t} Q\left(\xi_{x, n}\right)
$$

where $\xi_{x, n}=2 \pi n / L$ are the in-plane spatial frequencies of the periodic heating pattern with period $L, \eta_{0}$ is the modulation frequency, $\eta_{s}$ is the laser pulse repetition rate, and $t$ is the time delay of the probe relative to the pump. Here, $n$ and $m$ are integers. Equation (6) represents the timedomain TDTR signal as a double Fourier series, allowing us to observe that the transfer function is composed of a frequency response function, $H(\eta, \xi)$, evaluated at discrete temporal and spatial frequencies weighted by the Fourier series amplitudes of the heat source, $Q\left(\xi_{x, n}\right)$. The weights $Q\left(\xi_{x, n}\right)$ are given by the Fourier series components of the square wave, as shown in Fig. 1(b).

The inadequacy of the traditional model to explain the experiments implies that the surface-temperature response 
of the sample versus spatial and temporal frequency differs from that predicted by Fourier's law. To gain insight into this discrepancy, we calculate the surface-temperature response $H(\eta, \xi)$ as a function of temporal and in-plane spatial frequency of the applied surface heat flux for a thin film on substrate geometry with multidimensional heat conduction using the spectral BTE (see Sec. IIC). The amplitude and phase of the surface thermal response versus temporal frequency at different spatial frequencies computed from Fourier's law and the BTE are shown in Figs. 2(a) and 2(b). The calculations demonstrate that the BTE response agrees with the Fourier law calculation at smaller spatial frequencies but does not agree for larger spatial frequencies. As the poor fitting of Fig. 1(f) shows, the two fitting parameters of substrate thermal conductivity and interface conductance in the traditional model cannot explain these various differences at all of the temporal and spatial frequencies present in the TDTR signal. Therefore, a new model is needed to interpret the experiments.
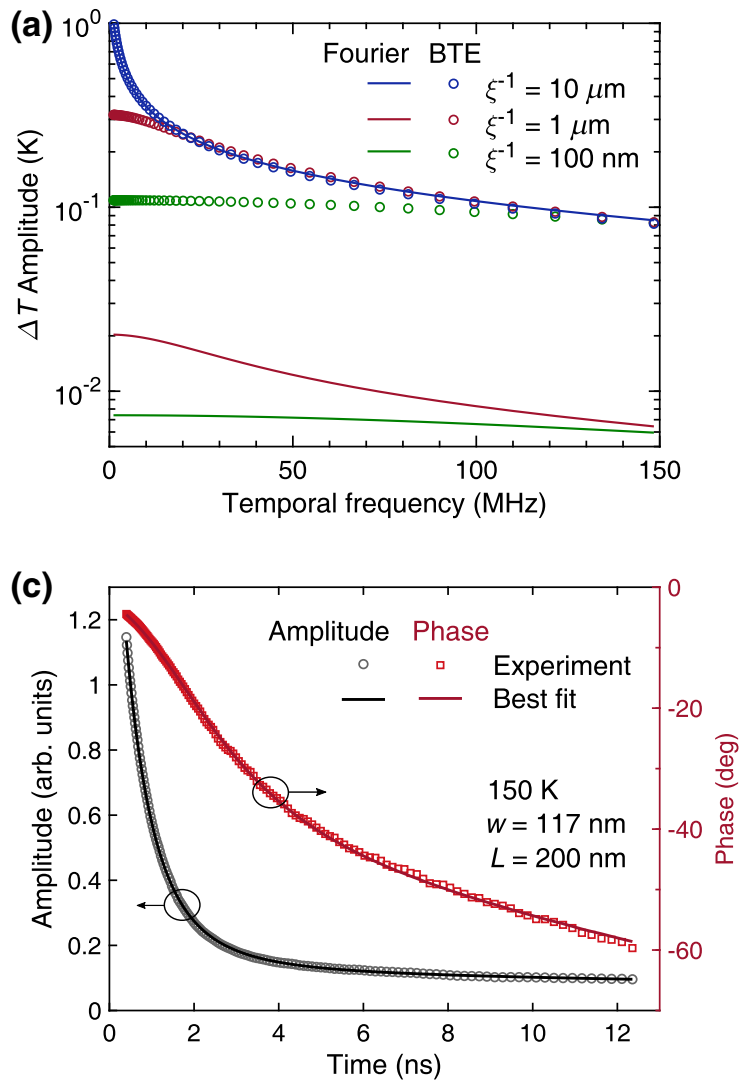

We introduce such a model by making several observations. In the weak quasiballistic regime where relevant timescales are far longer than phonon relaxation times, we have previously shown that apparent thermal properties can be rigorously defined [41], but these properties may not be constant with variations in spatial frequencies of the heater pattern. For instance, our analytical solution of the BTE for a semi-infinite domain shows that the thermal conductivity depends primarily on the magnitude of the spatial frequencies $\xi$ of the heating pattern rather than the temporal frequency [36], where $\xi=$ $\sqrt{\xi_{x}^{2}+\xi_{y}^{2}+\xi_{z}^{2}}$. In the line array pattern Fig. 1(a), $\xi_{x}$ corresponds to the in-plane direction. For the square-wave heater, $\xi_{x}=2 \pi n / L$, where $L$ is the period and $n$ is an integer, as shown in Fig. 1(b). $\xi_{y}=0$ due to the uniformity along the $y$ direction. The cross-plane spatial frequency $\xi_{z}$ is determined by the temporal heating frequencies in the modulated heating beam. For a given modulation frequency, the $\xi_{z}$ are fixed and, hence, our experiment
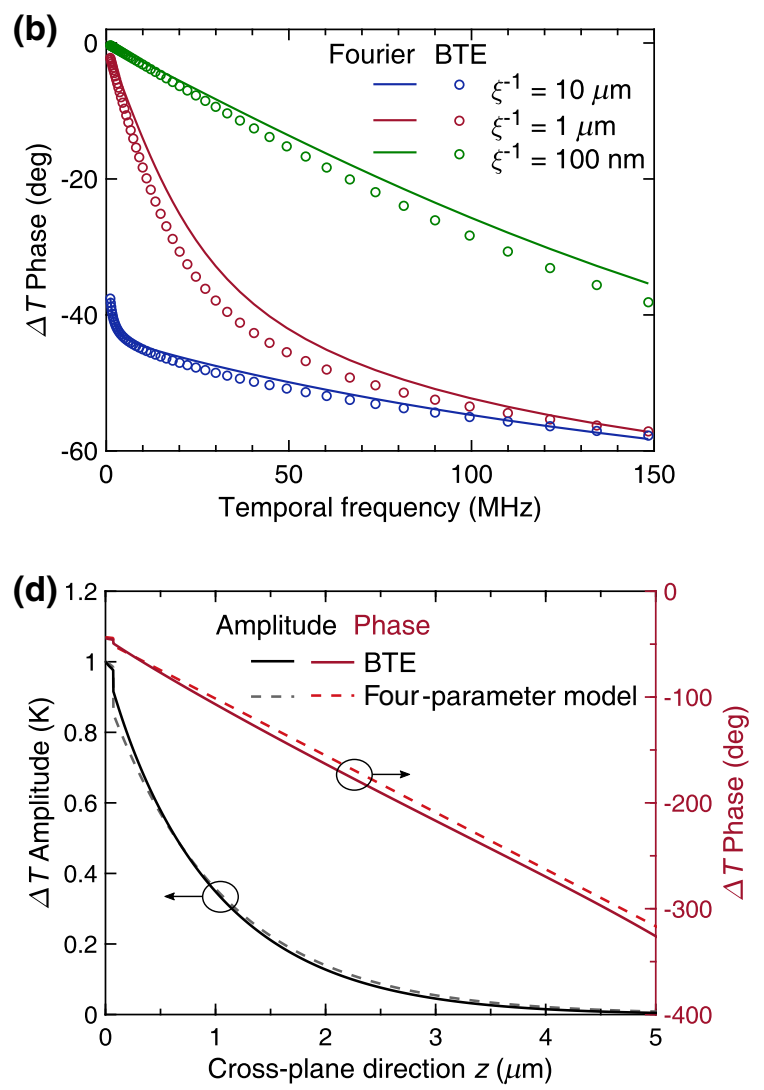

FIG. 2. Model to interpret TDTR data. (a) Amplitude and (b) phase of the thermal response of a thin film on a substrate versus temporal frequency for three spatial frequencies calculated using Fourier's law (lines) and BTE (symbols). (c) Refit of experimental data for the case in Fig. 1(f) with the four-parameter fitting model. The best fit gives an excellent fit to the data with $\kappa_{0}=142 \mathrm{~W} \mathrm{~m}{ }^{-1} \mathrm{~K}^{-1}$, $\kappa_{1}=4 \mathrm{~W} \mathrm{~m}^{-1} \mathrm{~K}^{-1}, G_{0}=137 \mathrm{MW} \mathrm{m}^{-2} \mathrm{~K}^{-1}, G_{1}=155 \mathrm{MW} \mathrm{m}^{-2} \mathrm{~K}^{-1}$, respectively. (d) Centerline temperature profile versus depth into the sample for a line array at a single temporal frequency of $10 \mathrm{MHz}$ calculated using the four-parameter model (dashed lines) and the exact solution from the BTE (solid lines). The four-parameter model reasonably explains the spatial temperature profile into the sample. 
primarily probes the effect of the in-plane spatial frequency $\xi_{x}$ on thermal conductivity set by the square-wave period. Additionally, we expect that the interface conductance will primarily depend on the cross-plane spatial frequencies $\xi_{z}$ that are, in turn, set by the temporal heating frequency (see Sec. 2 in the Supplemental Material [34]).

Given these observations, we construct a four-parameter model. Two parameters are thermal conductivities, one for zero spatial frequency $\kappa_{0}=\kappa\left(\xi_{x, 0}\right)$, corresponding to spatially uniform heating, and one for all higher-order spatial frequencies $\kappa_{1}=\kappa\left(\xi_{x,|n|>0}\right)$, where $\xi_{x,|n|>0}$ are integer multiples of the fundamental spatial frequency $\xi_{x, F}=2 \pi / L$. The other two are interface conductances, one at the modulation frequency $G_{0}=G\left(\eta_{0}\right)$ and one for higher-order temporal frequencies $G_{1}=G\left(\eta_{0}+m \eta_{s}\right)_{|m|>0}$. If the transport occurs by diffusion, thermal properties are constant for all spatial frequencies and $\kappa_{0}=\kappa_{1}$ and $G_{0}=G_{1}$. The model is not a two-channel model as has been reported in the past [26], but rather allows for the possibility that the spatial frequency components of the TDTR thermal response may be described by different thermal properties due to quasiballistic effects.

As in the traditional procedure, these parameters are obtained by fitting to the measured surface-temperature response of the sample. Applying the four-parameter model to the experimental data of Fig. 1(f) yields an excellent fit, as in Fig. 2(c). Of course, adding additional parameters to a model could lead to satisfactory fitting even if the underlying model is not physical. We provide support that our four-parameter model accurately describes the thermal transport in the system by computing the exact cross-plane spatial temperature profile in the sample using the BTE and comparing it to that obtained from fourparameter model with the best-fit parameters. The result is shown in Fig. 2(d) and demonstrates that the fourparameter model agrees nicely with the exact BTE result despite its simplicity, supporting the physical validity of our model. In the following discussion, we use these BTE simulations to compute TDTR data sets as a comparison with the experimental data; we term these simulations as synthetic data and the same four-parameter model is used to fit the synthetic data to obtain thermal conductivities and interface conductances exactly as in experiment.

\section{Results using four-parameter model}

We now apply this model to our measurements on nanolines. As the apparent thermal conductivity of the substrate is our primary interest, we defer discussion of interface conduction to Sec. 2 of the Supplemental Material [34]. When we measure the thermal conductivities $\left(\kappa_{0}\right.$ and $\left.\kappa_{1}\right)$ versus modulation frequency from 0.5 to $15 \mathrm{MHz}$, little dependence on modulation frequency is observed (see Sec. 3 and Fig. S3 in the Supplemental Material [34]), confirming our assumption that the measured thermal conductivity primarily depends on in-plane spatial frequency. For the measurements in all the figures below, the error bar indicates the standard deviation from measurements taken at multiple modulation frequencies between 1 and $10 \mathrm{MHz}$ and the uncertainty from fitting (see Secs. 4 and 5 in the Supplemental Material [34]), unless otherwise stated.

We first examine the thermal conductivities versus period with the duty cycle fixed at $50 \%$ at room temperature and $150 \mathrm{~K}$ in Fig. 3(a). The thermal conductivity at zero spatial frequency, $\kappa_{0}$, is independent of period and agrees with the bulk value of the thermal conductivity of sapphire at the relevant temperatures [39]. The thermal conductivity at higher spatial frequencies, $\kappa_{1}$, decreases with decreasing period for periods smaller than $2 \mu \mathrm{m}$ at room temperature. This observation indicates that phonon MFPs are on the scale of hundreds of nanometers and that the thermal resistance to heat flow at these spatial frequencies is larger than predicted by Fourier's law, in line with prior work [16]. At $150 \mathrm{~K}, \kappa_{1}$ is less than $\kappa_{0}$ as well as the bulk thermal conductivity even at period $3 \mu \mathrm{m}$, the maximum period used in the experiments, indicating that the mean free paths are longer than $3 \mu \mathrm{m}$ at $150 \mathrm{~K}$.

We present $\kappa_{1}$ versus period normalized to $\kappa_{0}$ at the specified temperatures in Fig. 3(b). The trend of increasing thermal conductivity with increasing period is reproduced by the synthetic TDTR data. Both the experimental and synthetic data show the physically intuitive result that the departure from the bulk thermal conductivity is larger at lower temperatures where phonon MFPs are longer than at room temperature.

While Figs. 3(a) and 3(b) show the relationship between $\kappa_{1}$ and period $L$ at a fixed duty cycle of $50 \%$, Fig. 3(c) shows how the $\kappa_{1}$ varies with fixed linewidth $w$ and changing period $L$. The figure shows that $\kappa_{1}$ increases as the period increases for a fixed linewidth of approximately 115 $\mathrm{nm}$ at different temperatures. Again, the synthetic TDTR data show a similar trend. In Fig. 3(d), at $150 \mathrm{~K}$, the $\kappa_{1}$ for different line array periods with a duty cycle of $67 \%$ are compared to those with a $50 \%$ duty cycle. For the same period, the patterns with a smaller duty cycle (smaller linewidth) have higher $\kappa_{1}$. Generally, these two figures indicate that $\kappa_{1}$ decreases as the lines become closer together.

\section{Role of the spatial frequency}

The observed trends of thermal conductivity $\kappa_{1}$ with geometrical properties of the heater pattern are difficult to interpret using the conventional notion that characteristic dimensions of the individual heaters are the key parameters that govern thermal transport. Under this assumption, the separation of the heater lines of a given width should have no impact on the thermal properties, yet a clear dependence is observed in Figs. 3(c) and 3(d). 
(a)

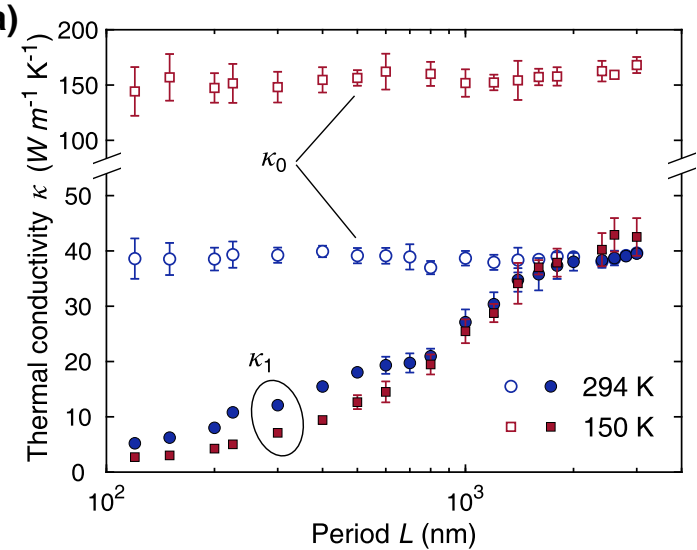

(c)

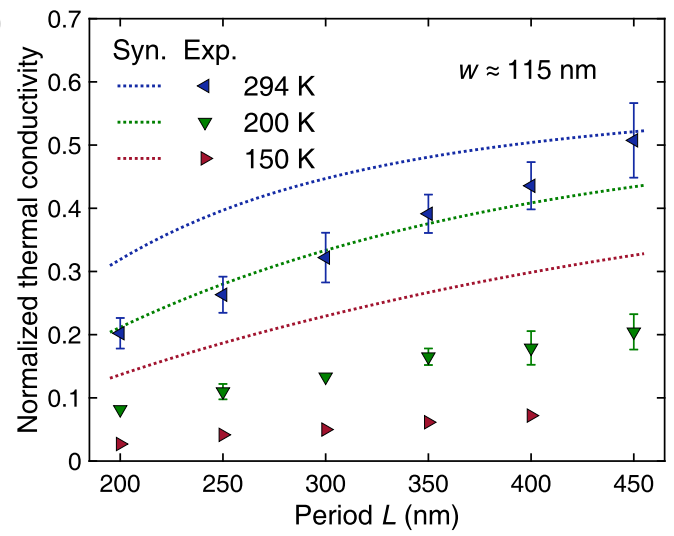

(b)

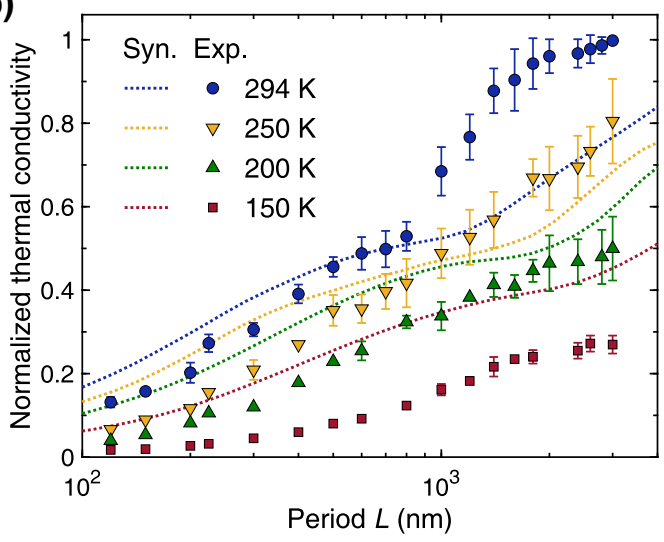

(d)

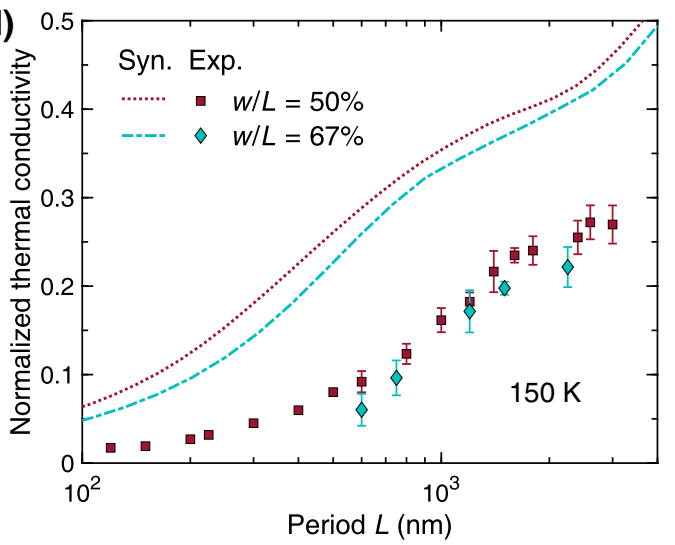

FIG. 3. Thermal conductivities of nanoline arrays. (a) Thermal conductivity $\kappa_{0}$ (open symbols) and $\kappa_{1}$ (filled symbols) versus period at $294 \mathrm{~K}$ (blue circles) and $150 \mathrm{~K}$ (red squares) for nanoline arrays with a $50 \%$ duty cycle. (b) $\kappa_{1}$ normalized to $\kappa_{0}$ versus period at different temperatures from measurements (symbols) and synthetic TDTR data (dotted lines). (c) $\kappa_{1}$ normalized to $\kappa_{0}$ from measurements (symbols) and synthetic TDTR data (dotted lines) versus period for nanoline arrays with $w \approx 115 \mathrm{~nm}$ at various temperatures. (d) $\kappa_{1}$ normalized to $\kappa_{0}$ versus period for duty cycles of $50 \%$ and $67 \%$, respectively. The temperature is $150 \mathrm{~K}$. In (c), the linewidth is fixed and the period changes, while in (d), the linewidth and period both change so as to keep the duty cycle fixed. In both (c) and (d), the synthetic TDTR data show similar trends. The synthetic data are the TDTR data sets computed from the BTE and then fit with the four-parameter model to obtain thermal conductivities and interface conductances.

These apparent inconsistencies can be eliminated by instead considering the spatial frequencies of the heating pattern as the key parameters. More precisely, we identify the key dimensionless parameter as $\xi_{x, F} \Lambda$, where $\Lambda$ is the phonon MFP and $\xi_{x, F}$ is the fundamental spatial frequency [36]. This parameter is very analogous to the familiar Knudsen number if the fundamental spatial frequency is written as $2 \pi / L$.

Using this dimensionless parameter, the observed trends can be explained simply by considering the spatial frequencies of the heater pattern. For instance, the trend of increasing $\kappa_{1}$ with increasing period, with duty cycle held constant at $50 \%$ as in Fig. 3(b), can be explained by recognizing that an increasing period $L$ implies a decreasing $\xi_{x, F}$ and thermal conductivity increases with decreasing spatial frequency $[36,42]$. More subtle trends such as the increase of $\kappa_{1}$ with period for a fixed linewidth in Fig. 3(c) can also be rationalized by the same explanation. The dependence of $\kappa_{1}$ on duty cycle in Fig. 3(d) reflects the dependence of the overall thermal response on the relative weights of the nonzero in-plane spatial frequencies in the heating pattern. In this case, a duty cycle of $67 \%$ has larger weights on higher spatial frequencies than the $50 \%$ duty cycle, resulting in a slightly lower thermal conductivity. However, our measurements show that the primary parameter that governs $\kappa_{1}$ is the fundamental spatial frequency set by the period of the heating pattern.

To further demonstrate the importance of the fundamental spatial frequency, in Fig. 4, we plot all of our data on nanoline arrays versus the fundamental spatial frequency of the heating pattern. At each temperature, the data nicely collapse onto a single curve that decreases monotonically as the fundamental spatial frequency increases, exactly as predicted by theory [36]. The trend is reproduced by the synthetic TDTR data for line arrays with a 50\% duty cycle. 


\section{HEAT DISSIPATION FOR DIFFERENT HEATER GEOMETRIES}

The identification of the critical role of the spatial frequency allows us to examine several subtle points regarding quasiballistic thermal transport from nanoscale heat sources. First, the conventional view holds that as an individual heat source, either isolated or in a pattern, becomes far smaller than mean free paths, a local hot spot will form at the heater due to the ballistic thermal resistance. However, as qualitatively suggested previously [30], this argument fails to account for the lack of in-plane gradient if heat sources in a pattern are sufficiently close together. In this situation, the thermal transport that occurs will be identical to that from a continuous thin film heater.

We experimentally examine this prediction by considering a qualitative measure of the thermal resistance as an apparent substrate thermal conductivity obtained by fitting the amplitude component of the TDTR signal using the model of Ref. [16]. We perform this fitting for nanoline arrays of variable period in Fig. 5(a). We find that the apparent thermal conductivity with the nanoline array obtained experimentally has only weak dependence on the period, indicating that the thermal resistance of the substrate has little dependence on the in-plane thermal gradient. This weak period dependence is also observed in the synthetic TDTR data. Hence, the heater pattern conducts heat nearly identically as would a continuous thin film.

Analysis of the spatial frequencies of the nanoline heater pattern allows us to explain this observation. As shown in Fig. 1(b), the nanoline heater pattern can be expressed as a Fourier series of discrete spatial frequencies and corresponding weights, and the overall thermal response depends on these weights and the relative magnitudes of the thermal responses at the discrete spatial frequencies. Importantly, the thermal response at zero spatial frequency makes the largest contribution to the overall thermal response and, by definition, it is unaffected by the linewidth of the heating pattern, no matter how small. At higher spatial frequencies, the thermal response amplitude decreases dramatically with increasing spatial frequency. Additionally, the weights only depend on the duty cycle of the pattern and also decrease with increasing spatial frequency. The weak dependence of the nanoline apparent thermal conductivity on period can thus be understood from the relatively small contribution of thermal responses at higher spatial frequencies compared to that at zero spatial frequency (dc). Therefore, considering the superposition of thermal responses shows that the overall thermal response of the nanoline pattern will be only weakly affected by its geometry, exactly as observed in experiment. This dependence will weaken as the linewidth decreases and hence the fundamental spatial frequency increases.

On the other hand, consider a Gaussian spot heater created by a focused laser beam. As shown in Fig. 5(a), in this

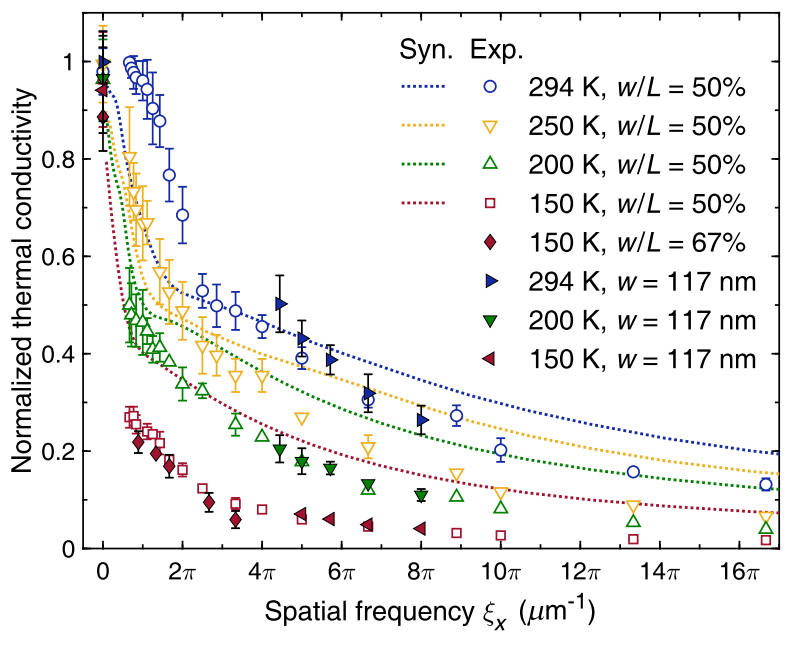

FIG. 4. Normalized thermal conductivity versus spatial frequency. $\kappa_{1}$ normalized to $\kappa_{0}$ at each temperature versus spatial frequency at different temperatures from measurements (open symbols) and synthetic TDTR data (dotted lines) for line arrays with a $50 \%$ duty cycle. Measurements for line arrays with other duty cycles are also shown with filled symbols. For all the measurements, the thermal conductivity decreases monotonically as the spatial frequency increases. At a given temperature, the duty cycle has only a little effect on the thermal conductivity for a given spatial frequency, indicating that spatial frequency is the key parameter. The synthetic TDTR data show similar trends as those observed experimentally.

situation, we observe an obvious decrease in apparent thermal conductivity at characteristic length scales far larger than those achieved in the nanoline arrays. Again, this trend is reproduced by synthetic TDTR data. To understand this observation, we must examine the overall thermal response to the Gaussian heater, given as

$$
Z(t)=\int_{0}^{\infty} \sum_{m=-\infty}^{\infty} H\left(\eta+m \eta_{s}, \xi_{r}\right) e^{i m \eta_{s} t} Q\left(\xi_{r}\right) \xi_{r} d \xi_{r},
$$

where $\xi_{r}^{2}=\xi_{x}^{2}+\xi_{y}^{2}$ is the in-plane spatial Fourier variable in polar coordinates and $Q\left(\xi_{r}\right)=\exp \left[-\left(r_{0}^{2}+r_{1}^{2}\right) \xi_{r}^{2} / 8\right]$, where $r_{0}$ and $r_{1}$ are the $1 / e^{2}$ radii of the pump and probe beams, respectively. At a given $\xi_{r}$, the overall thermal response, as shown in Fig. 5(b), is weighted by $Q\left(\xi_{r}\right) \xi_{r}$. Because of the factor of $\xi_{r}$ in the weights, the dc component $\left(\xi_{r}=0\right)$ contributes nothing to the overall response and the response peaks at a nonzero $\xi_{r}$, at which the deviation from the Fourier response may be prominent. Therefore, for an isolated heater localized in more than one spatial dimension, heat dissipation can be substantially impeded compared to the predictions of Fourier's law, in line with prior work $[15,19,30,43]$. This ballistic resistance can be mitigated by placing heaters sufficiently close such that the dc component once again contributes to the thermal response. 
(a)

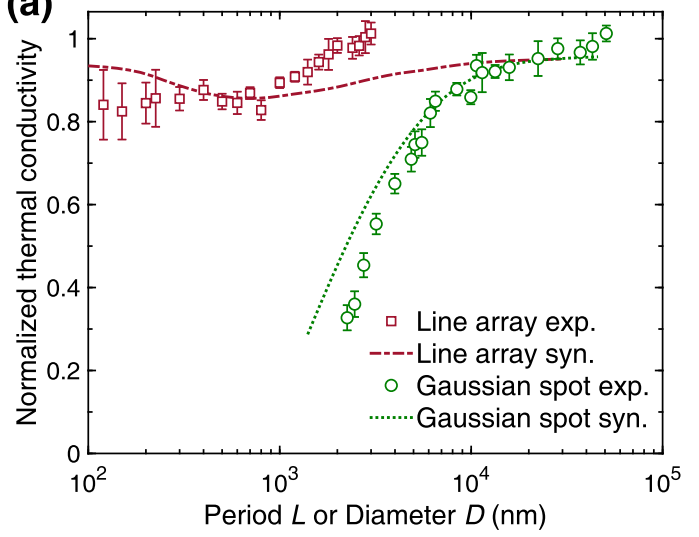

(b)

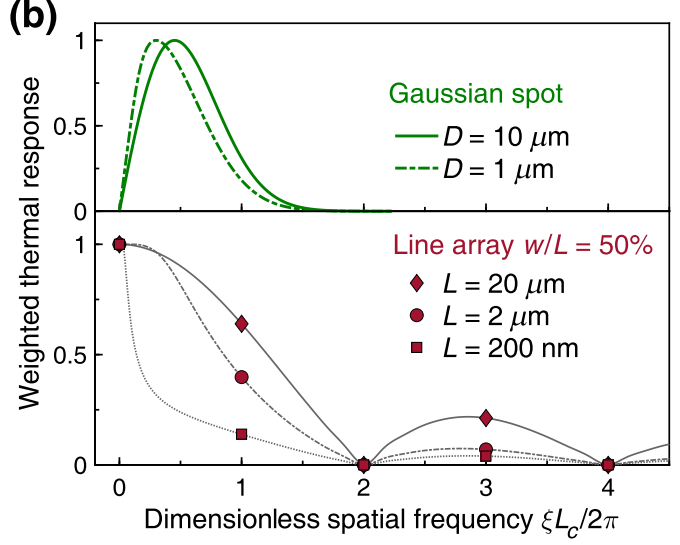

FIG. 5. Heat dissipation for different heater patterns. (a) Apparent thermal conductivity of the nanoline array with a 50\% duty cycle and a Gaussian spot versus characteristic size from experiments and synthetic TDTR data normalized by $\kappa_{0}$. The thermal conductivity strongly decreases as the Gaussian diameter decreases, but only weak dependence is observed for the nanoline array. (b) Weighted thermal response versus dimensionless spatial frequency, at 5-MHz heating frequency for a Gaussian spot (top) and the nanoline array (bottom, red symbols). The grey lines show the continuous function from which the discrete points for the nanoline array are obtained. The zero spatial frequency response dominates the overall response for the line array, while it is zero for the Gaussian heating pattern due to the weighting factor of $\xi_{r}$. The absence of the dc response is the reason that quasiballistic effects are readily observed with a Gaussian spot. $L_{c}$ is the characteristic length used to normalize the spatial frequency, which is the period and root-mean square of the pump and probe $1 / e^{2}$ diameters for the nanoline array and Gaussian spot, respectively. Note that the $x$ axis is the dimensionless spatial frequency and that patterns with smaller $L_{c}$ have larger fundamental spatial frequency.

These results show that measurements performed with different heater patterns can yield qualitatively different results, even if they are performed on the same material with the same characteristic heater size. For instance, Hu et al. [16] reported TDTR measurements on lithographically patterned dot arrays, observing substantial deviations from Fourier's law. The difference between their observations and ours could be due to their use of 2D heater dots rather than 1D heater lines, leading to correspondingly lower dc weights in the thermal response.

In addition to the importance of the spatial frequencies of the heating pattern, our analysis also shows the importance of the spatial frequencies in the probing pattern. In our study, these two sets of spatial frequencies are the same. However, in other experiments, the two may differ. For instance, the measurements of Johnson et al. [25], Siemens et al. [14], and Hoogeboom-Pot et al. [20] measure diffraction from the heating pattern and, hence, select only the first harmonic of the spatial frequency to measure. In our terminology, these measurements closely correspond to obtaining $\kappa_{1}$ only. Hence, measurements that employ diffraction for probing may observe large size effects for nanoline heater patterns [14,20], while thermoreflectance experiments such as those reported here observe only weak size effects. The importance of the probing pattern has been previously recognized [19,44]; our work allows these effects to be explained simply in terms of the spatial frequencies and weights of the probing pattern.

We finally note that, although obtaining physical information about phonons from these data, such as their mean free paths, is highly desirable, doing so is not so simple. As we have shown previously [37], the TDTR signal also depends on the spectrum of injected phonons. In this work, we mainly focus on understanding trends in thermal conductivity as cross-plane effects are fixed and inplane spatial frequencies are systematically varied. A full description of the transport requires a full BTE solution that we will address in a future work.

Finally, we discuss the impacts of our study on applications. Our work has demonstrated that the effective thermal resistance of a substrate in the quasiballistic regime depends on the spatial frequencies of the heating pattern rather than the geometrical properties of individual heaters. This result has an important impact on strategies to enhance thermal dissipation in microelectronic devices [9], for example. While the traditional assumption has been that nanoscale devices experience increased local heating due to their small size, our work shows that if these individual devices are arranged periodically with sufficiently high density, the thermal resistance decreases toward the bulk limit. In other words, closely packing individual devices can actually lead to decreased thermal resistance even if the devices' dimensions are much smaller than phonon mean free paths. Our work provides a means to quantitatively determine the effective thermal resistance of a substrate for an arbitrary heater pattern on a surface simply by considering the spatial frequencies of the heating pattern.

In summary, we have investigated quasiballistic thermal transport from nanoline array heaters. Our work experimentally establishes the central role of the spatial 
frequency content of the heater pattern in understanding and manipulating heat transport in the quasiballistic regime. Unintuitive trends of apparent thermal conductivity with heater pattern geometry are easily interpreted by examining the spatial frequencies of the heater pattern. Our work also demonstrates that different heater and probing geometries may have very different thermal responses, even if the spatial frequencies are comparable, due to the different weights of spatial frequencies in the overall thermal response and those selected for measurement. Our work provides the insight needed to rationally enhance thermal dissipation in applications such as thermal management of microelectronics.

\section{ACKNOWLEDGMENTS}

The authors thank Alexei Maznev for valuable comments and discussions; Lucas Lindsay for providing the first-principles calculations for silicon; the Kavli Nanoscience (KNI) at Caltech for the availability of critical cleanroom facilities; Guy A. DeRose for discussions and comments on the nanoline array fabrications; Matt H. Sullivan for assistance on FIB and e-beam lithography processing; Carol M. Carland for TEM assistance; and Bo Sun and Peishi Cheng for proofreading the article. This work was sponsored in part by the National Science Foundation under Grant No. CBET CAREER 1254213 and by Boeing under the Boeing-Caltech Strategic Research \& Development Relationship Agreement. H.Z. also gratefully acknowledges the financial support of the CAS Pioneer Hundred Talents Program.

The experiments, analytical model, and simulations were conceived by A.J.M. The experiments and fabrication were performed by X.C. The simulations were performed by C.H. and N.K.R. H.Z. initialized the nanoline array fabrications. The manuscript was written by X.C. and A.J.M. with comments and input from all authors. X.C. and C.H. contributed equally to this work.

[1] B. Poudel, Q. Hao, Y. Ma, Y. Lan, A. Minnich, B. Yu, X. Yan, D. Wang, A. Muto, D. Vashaee, X. Chen, J. Liu, M. S. Dresselhaus, G. Chen, and Z. R. Ren, High-thermoelectric performance of nanostructured bismuth antimony telluride bulk alloys, Science 320, 634 (2008).

[2] G. J. Snyder and E. S. Toberer, Complex thermoelectric materials, Nat. Mater. 7, 105 (2008).

[3] K. Biswas, J. He, I. D. Blum, C.-I. Wu, T. P. Hogan, D. N. Seidman, V. P. Dravid, and M. G. Kanatzidis, Highperformance bulk thermoelectrics with all-scale hierarchical architectures, Nature 489, 414 (2012).

[4] R. J. Mehta, Y. Zhang, C. Karthik, B. Singh, R. W. Siegel, T. Borca-Tasciuc, and G. Ramanath, A new class of doped nanobulk high-figure-of-merit thermoelectrics by scalable bottom-up assembly, Nat. Mater. 11, 233 (2012).
[5] M. Zebarjadi, K. Esfarjani, M. Dresselhaus, Z. Ren, and G. Chen, Perspectives on thermoelectrics: From fundamentals to device applications, Energy Environ. Sci. 5, 5147 (2012).

[6] D. G. Cahill, P. V. Braun, G. Chen, D. R. Clarke, S. Fan, K. E. Goodson, P. Keblinski, W. P. King, G. D. Mahan, A. Majumdar, H. J. Maris, S. R. Phillpot, E. Pop, and L. Shi, Nanoscale thermal transport. II. 2003-2012, Appl. Phys. Rev. 1, 011305 (2014).

[7] E. Pop, Energy dissipation and transport in nanoscale devices, Nano Res. 3, 147 (2010).

[8] A. L. Moore and L. Shi, Emerging challenges and materials for thermal management of electronics, Mater. Today 17, 163 (2014).

[9] L. Shi, C. Dames, J. R. Lukes, P. Reddy, J. Duda, D. G. Cahill, J. Lee, A. Marconnet, K. E. Goodson, J.-H. Bahk, A. Shakouri, R. S. Prasher, J. Felts, W. P. King, B. Han, and J. C. Bischof, Evaluating broader impacts of nanoscale thermal transport research, Nanoscale Microscale Thermophys. Eng. 19, 127 (2015).

[10] E. Lee, T. Zhang, T. Yoo, Z. Guo, and T. Luo, Nanostructures significantly enhance thermal transport across solid interfaces, ACS Appl. Mater. Interfaces 8, 35505 (2016).

[11] T. Klitsner, J. VanCleve, H. E. Fischer, and R. Pohl, Phonon radiative heat transfer and surface scattering, Phys. Rev. B 38, 7576 (1988).

[12] A. Minnich, Advances in the measurement and computation of thermal phonon transport properties, J. Phys.: Condens. Matter 27, 053202 (2015).

[13] Y. K. Koh and D. G. Cahill, Frequency dependence of the thermal conductivity of semiconductor alloys, Phys. Rev. B 76, 075207 (2007)

[14] M. E. Siemens, Q. Li, R. Yang, K. A. Nelson, E. H. Anderson, M. M. Murnane, and H. C. Kapteyn, Quasi-ballistic thermal transport from nanoscale interfaces observed using ultrafast coherent soft X-ray beams, Nat. Mater. 9, 26 (2010).

[15] A. J. Minnich, J. A. Johnson, A. J. Schmidt, K. Esfarjani, M. S. Dresselhaus, K. A. Nelson, and G. Chen, Thermal conductivity spectroscopy technique to measure phonon mean free paths, Phys. Rev. Lett. 107, 095901 (2011).

[16] Y. Hu, L. Zeng, A. J. Minnich, M. S. Dresselhaus, and G. Chen, Spectral mapping of thermal conductivity through nanoscale ballistic transport, Nat. Nanotechnol. 10, 701 (2015).

[17] L. Zeng, K. C. Collins, Y. Hu, M. N. Luckyanova, A. A. Maznev, S. Huberman, V. Chiloyan, J. Zhou, X. Huang, K. A. Nelson, and G. Chen, Measuring phonon mean free path distributions by probing quasiballistic phonon transport in grating nanostructures, Sci. Rep. 5, 17131 (2015).

[18] K. T. Regner, D. P. Sellan, Z. Su, C. H. Amon, A. J. McGaughey, and J. A. Malen, Broadband phonon mean free path contributions to thermal conductivity measured using frequency domain thermoreflectance, Nat. Commun. 4, 1640 (2013).

[19] R. B. Wilson and D. G. Cahill, Anisotropic failure of fourier theory in time-domain thermoreflectance experiments, Nat. Commun. 5, 5075 (2014).

[20] K. M. Hoogeboom-Pot, J. N. Hernandez-Charpak, X. Gu, T. D. Frazer, E. H. Anderson, W. Chao, R. W. Falcone, R. Yang, M. M. Murnane, and H. C. Kapteyn, A new 
regime of nanoscale thermal transport: Collective diffusion increases dissipation efficiency, Proc. Natl. Acad. Sci. USA 112, 4846 (2015).

[21] B. Vermeersch, J. Carrete, N. Mingo, and A. Shakouri, Superdiffusive heat conduction in semiconductor alloys. I. Theoretical foundations, Phys. Rev. B 91, 085202 (2015).

[22] B. Vermeersch, A. M. Mohammed, G. Pernot, Y. R. Koh, and A. Shakouri, Superdiffusive heat conduction in semiconductor alloys. II. Truncated Lévy formalism for experimental analysis, Phys. Rev. B 91, 085203 (2015).

[23] F. Yang and C. Dames, Heating-frequency-dependent thermal conductivity: An analytical solution from diffusive to ballistic regime and its relevance to phonon scattering measurements, Phys. Rev. B 91, 165311 (2015).

[24] K. Esfarjani, G. Chen, and H. T. Stokes, Heat transport in silicon from first-principles calculations, Phys. Rev. B 84, 085204 (2011).

[25] J. A. Johnson, A. Maznev, J. Cuffe, J. K. Eliason, A. J. Minnich, T. Kehoe, C. M. S. Torres, G. Chen, and K. A. Nelson, Direct measurement of room-temperature nondiffusive thermal transport over micron distances in a silicon membrane, Phys. Rev. Lett. 110, 025901 (2013).

[26] R. Wilson, J. P. Feser, G. T. Hohensee, and D. G. Cahill, Two-channel model for nonequilibrium thermal transport in pump-probe experiments, Phys. Rev. B 88, 144305 (2013).

[27] Y. K. Koh, D. G. Cahill, and B. Sun, Nonlocal theory for heat transport at high frequencies, Phys. Rev. B 90, 205412 (2014).

[28] K. Regner, A. J. McGaughey, and J. A. Malen, Analytical interpretation of nondiffusive phonon transport in thermoreflectance thermal conductivity measurements, Phys. Rev. B 90, 064302 (2014).

[29] D. Ding, X. Chen, and A. J. Minnich, Radial quasiballistic transport in time-domain thermoreflectance studied using Monte Carlo simulations, Appl. Phys. Lett. 104, 143104 (2014).

[30] R. Wilson and D. G. Cahill, Limits to fourier theory in high thermal conductivity single crystals, Appl. Phys. Lett. 107, 203112 (2015).

[31] K. Kang, Y. K. Koh, C. Chiritescu, X. Zheng, and D. G. Cahill, Two-tint pump-probe measurements using a femtosecond laser oscillator and sharp-edged optical filters, Rev. Sci. Instrum. 79, 114901 (2008).

[32] D. G. Cahill, Analysis of heat flow in layered structures for time-domain thermoreflectance, Rev. Sci. Instrum. 75, 5119 (2004).
[33] A. J. Schmidt, X. Chen, and G. Chen, Pulse accumulation, radial heat conduction, and anisotropic thermal conductivity in pump-probe transient thermoreflectance, Rev. Sci. Instrum. 79, 114902 (2008).

[34] See Supplemental Material at http://link.aps.org/supple mental/10.1103/PhysRevApplied.10.054068, which includes Refs. [16,33,44]. Detailed information includes fitting of experimental and synthetic TDTR data, interface conductance of nanoline array, modulation frequency dependence of thermal conductivities, sensitivity and uncertainty analysis, determining linewidth of the patterns, reproducibility on different samples, and effect of thermoreflectance of sapphire substrate.

[35] C. Hua and A. J. Minnich, Semi-analytical solution to the frequency-dependent Boltzmann transport equation for cross-plane heat conduction in thin films, J. Appl. Phys. 117, 175306 (2015).

[36] C. Hua and A. J. Minnich, Analytical Green's function of the multidimensional frequency-dependent phonon Boltzmann equation, Phys. Rev. B 90, 214306 (2014).

[37] C. Hua, X. Chen, N. K. Ravichandran, and A. J. Minnich, Experimental metrology to obtain thermal phonon transmission coefficients at solid interfaces, Phys. Rev. B 95, 205423 (2017).

[38] L. Lindsay, D. A. Broido, and T. L. Reinecke, Ab initio thermal transport in compound semiconductors, Phys. Rev. B 87, 165201 (2013).

[39] G. A. Slack, Thermal conductivity of $\mathrm{MgO}, \mathrm{Al}_{2} \mathrm{O}_{3}$, $\mathrm{MgAl}_{2} \mathrm{O}_{4}$, and $\mathrm{Fe}_{3} \mathrm{O}_{4}$ Crystals from $3^{\circ}$ to $300^{\circ} \mathrm{K}$, Phys. Rev. 126, 427 (1962).

[40] D. G. Cahill, S. Lee, and T. I. Selinder, Thermal conductivity of $\kappa-\mathrm{Al}_{2} \mathrm{O}_{3}$ and $\alpha-\mathrm{Al}_{2} \mathrm{O}_{3}$ wear-resistant coatings, J. Appl. Phys. 83, 5783 (1998).

[41] C. Hua and A. J. Minnich, Transport regimes in quasiballistic heat conduction, Phys. Rev. B 89, 094302 (2014).

[42] A. J. Minnich, Multidimensional quasiballistic thermal transport in transient grating spectroscopy, Phys. Rev. B 92, 085203 (2015).

[43] Y. Ju and K. Goodson, Phonon scattering in silicon films with thickness of order $100 \mathrm{~nm}$, Appl. Phys. Lett. 74, 3005 (1999).

[44] L. Zeng and G. Chen, Disparate quasiballistic heat conduction regimes from periodic heat sources on a substrate, J. Appl. Phys. 116, 064307 (2014). 